\title{
Finite Temperature Phase Behavior of Viral Capsids as Oriented Particle Shells
}

\author{
Amit R. Singh $\odot,{ }^{1,2}$ Andrej Košmrlj $\oplus^{3,4}$ and Robijn Bruinsma $\oplus^{5}$ \\ ${ }^{1}$ Department of Physics and Astronomy, Johns Hopkins University, Baltimore, Maryland 21218, USA \\ ${ }^{2}$ Currently at Department of Mechanical Engineering, Birla Institute of Technology and Science, Pilani, Rajasthan 333031, India \\ ${ }^{3}$ Department of Mechanical and Aerospace Engineering, Princeton University, Princeton, New Jersey 08540, USA \\ ${ }^{4}$ Princeton Institute for the Science and Technology of Materials (PRISM), Princeton University, Princeton, New Jersey 08544, USA \\ ${ }^{5}$ Departments of Physics and Astronomy, and Chemistry and Biochemistry, University of California, \\ Los Angeles, California 90095, USA
}

(Received 19 November 2019; accepted 16 March 2020; published 13 April 2020; corrected 17 April 2020)

\begin{abstract}
A general phase plot is proposed for discrete particle shells that allows for thermal fluctuations of the shell geometry and of the inter-particle connectivities. The phase plot contains a first-order melting transition, a buckling transition, and a collapse transition and is used to interpret the thermodynamics of microbiological shells.
\end{abstract}

DOI: 10.1103/PhysRevLett.124.158101

The development of shells that protect microbiological systems from a hostile environment yet still allow for exchange of key nutrients was an essential step in the evolution of life [1]. These shells are composed of molecules decorated with both hydrophobic and hydrophilic groups ("amphiphiles") in such a way that in an aqueous environment they assemble into closed, semipermeable shells. An important example are the amphiphilic protein shells that surround viruses [2] as well as many bacteria and most archaea [3]. Cryogenic-based microscopy studies [2] had indicated that these "capsids" in general are strictly organized, crystallographic structures (usually icosahedral or helical) [4], but this view is being challenged. Thermodynamic studies of viral self-assembly indicate, for the case of the assembly of viruses in solutions with higher protein concentrations, that the interaction energies between capsid proteins ("subunits") should be no more than a few times the thermal energy at room temperature in order to avoid the production of malformed capsids [5-8]. Finite temperature studies also showed that, due to thermal fluctuations, at least some viral capsids are dynamical in nature and that the dynamics plays a role in the life-cycle of the virus $[10,11]$. Some capsids are even in a molten or "pleomorphic" state [12,13]. Finally, all-atom molecular dynamics (MD) simulations of capsids revealed that they can collapse under the action of thermal fluctuations [19].

The study of the melting and thermal collapse of a shell with a limited number of constituent components $\left(10^{2}-10^{3}\right)$ is an interesting statistical physics problem in its own right. The geometry of the shell over which the components are moving itself is defined by the position vectors of these same particles and hence subject to thermal fluctuations [20]. Here, we propose a generic phase diagram for the melting and collapse of discrete shells obtained by comparing MD simulations of a coarse-grained model of capsids with the continuum theory of thermally fluctuating surfaces.

We first discuss the MD simulations. The coarse-grained model is based on the so-called oriented particle system (or "OPS") [21]. An OPS is defined as a cluster of $N$ orientable and interacting point particles located at $\mathbf{r}_{i}$. An orientationdependent pair interaction $V\left(\mathbf{r}_{i}, \mathbf{n}_{i} ; \mathbf{r}_{j}, \mathbf{n}_{j}\right)$ acts between particle pairs $i$ and $j$ with a separation vector $\mathbf{r}_{i j}=\mathbf{r}_{i}-\mathbf{r}_{j}$ and unit vectors $\mathbf{n}_{i}$ and $\mathbf{n}_{j}$ describing their orientations [see Fig. 1 (left)]. The oriented pair interaction used in the simulations was

$$
\begin{aligned}
V\left(\mathbf{r}_{i}, \mathbf{n}_{i} ; \mathbf{r}_{j}, \mathbf{n}_{j}\right)= & V_{m}\left(1-e^{-\alpha\left(\left|\mathbf{r}_{i j}\right|-a\right)}\right)^{2} \\
& +K\left|\mathbf{n}_{i}-\mathbf{n}_{j}\right|^{2}+K\left[\left(\mathbf{n}_{i}+\mathbf{n}_{j}\right) \hat{\mathbf{r}}_{i j}\right]^{2} .
\end{aligned}
$$

The first term is the Morse pair interaction [22], with binding energy $V_{m}$, equilibrium bond distance $a$, and potential well width $1 / \alpha$. The second and third terms are

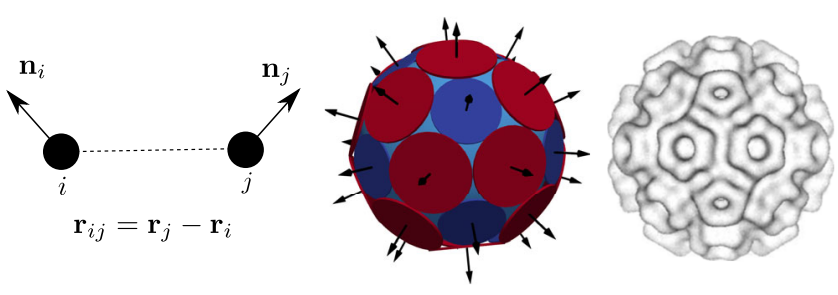

FIG. 1. Left: oriented particles $i$ and $j$ are separated by $\mathbf{r}_{i j}=$ $\mathbf{r}_{i}-\mathbf{r}_{j}$ with orientations indicated by the unit vectors $\mathbf{n}_{i}$ and $\mathbf{n}_{j}$. Middle: the icosahedral groundstate configuration of an $N=32$ oriented particle system. The particle positions are displayed as the centers of close-packed disks. The orientations are displayed as the normals to the disks. Right: micrograph of the Cowpea Chlorotic Mottle virus [23] with 32 capsomers. 
known as the "conormality" and "cocircularity" terms of an OPS system. Together, these two terms are minimized if the two particles have the same orientation and if that shared orientation is perpendicular to the unit vector $\hat{\mathbf{r}}_{i j}$ that is directed along the separation vector. Only interactions between particles that are nearest neighbors are included, where the set of nearest neighbors can change over time due to thermal fluctuations.

An OPS can be viewed as a coarse-grained representation of a viral capsid by having the particle locations correspond to the centers of the "capsomers" of viral capsids. The latter are disklike groups of either six or five subunits that frequently act as the basic building blocks of a capsid $[4,24]$. The orientational degrees of freedom correspond to the normals to the capsomers, the depth $V_{m}$ of the Morse potential to the capsomer binding energy (a few $k_{B} T$ $[25,26])$, and the length scale $a$ to the diameter of a capsomer (a few nanometers [2]). Because the range of the hydrophobic attraction between capsomers is short compared to their diameter, the dimensionless parameter $\alpha a$ characterizing the width of the Morse potential needs to be significantly larger than one. We used $\alpha a \approx 4.621$. Next, $K$ is a measure of the bending stiffness of the shell (estimated to be in the range of $10^{2} k_{B} T[27,28]$ ). Finally, because a large energy penalty is known to obstruct the removal of single capsomers from assembled shells [29], evaporation of particles from the OPS shell is suppressed by a soft fixed-area constraint imposed via the augmented Lagrange multiplier method (see [30], Sec. I).

Figure 1 (middle) shows the minimum energy state of an $N=32$ OPS for the case that $K / V_{m}$ is large compared to one. The shell has icosahedral symmetry with the twelve blue disks indicating the five-fold symmetry sites of the icosahedron (for actual capsids, these disks would correspond to pentameric protein capsomers while the remaining 20 red disks would correspond to hexameric capsomers). This structure should be compared to that of the " $T=3$ " icosahedral pattern [4] of the 32 capsomers of the capsid of the Cowpea Chlorotic Mottle virus [23] shown in the right of Fig. 1.

Next, we carried out Brownian Dynamics simulations of $N=72$ OPS systems using computational methods discussed further in the Supplemental Material [30] (Sec. I). The phase behavior was determined in terms of the two thermodynamic parameters $\beta^{-1}=k_{B} T / V_{m}$, a dimensionless measure of temperature in units of the depth $V_{m}$ of the pair interaction, and $\gamma=2 \alpha^{2} V_{m} R^{2} /(3 K)$ a dimensionless measure of the inverse of the bending stiffness $K$ ( $R \sim a N^{1 / 2}$ is the shell radius). In continuum theory $\gamma$ is known as the Föppl-von Kármán (FvK) number [39]. For different values of these two parameters we encountered ordered, molten, buckled, and collapsing shells. Representative realizations are shown in Figs. 2 and 3.

The degree of fluidity of a shell was monitored using a dynamical method based on plots of the mean square of the
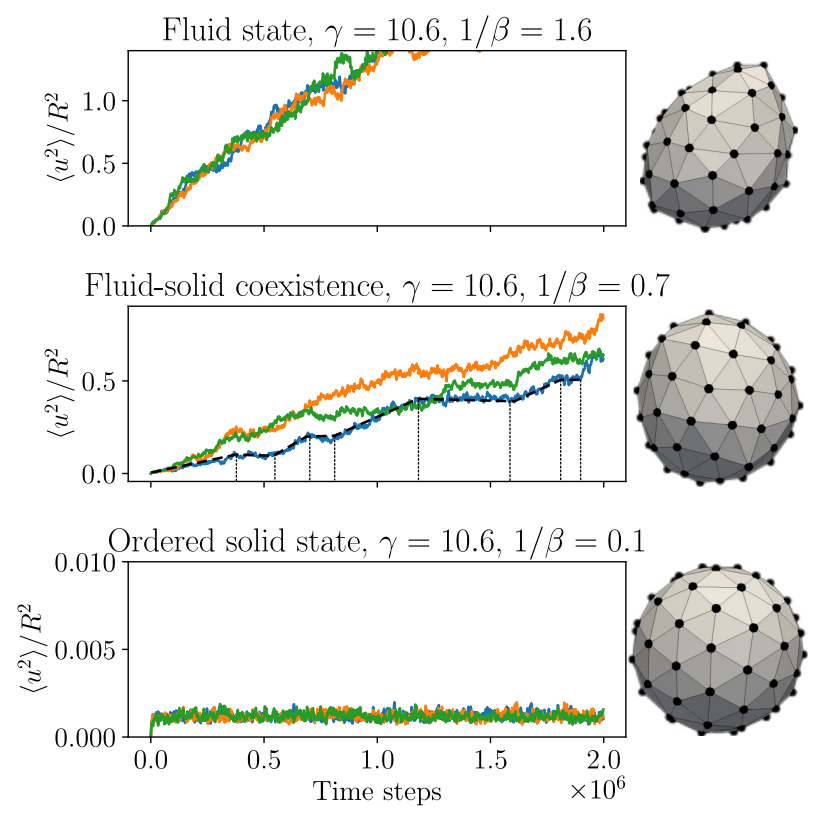

FIG. 2. Plots of the mean square $\left\langle u^{2}(t)\right\rangle$ of the particle displacements as a function of time for FvK number $\gamma=10.6$ and three different temperatures $\beta^{-1}$. Three separate simulation runs are shown in each case (orange, blue, and green respectively). Bottom: for low temperatures $\left(\beta^{-1}=0.1\right),\left\langle u^{2}(t)\right\rangle$ reaches a constant value after a short transient (ordered solid state). Top: for high temperatures $\left(\beta^{-1}=1.6\right),\left\langle u^{2}(t)\right\rangle$ is proportional to time for the three runs, indicating diffusion (fluid state). Middle: for intermediate temperatures $\left(\beta^{-1}=0.7\right)$ the mean square displacements curves alternate between intervals where $\left\langle u^{2}(t)\right\rangle$ steadily increases in time and intervals where it is roughly constant (fluid-solid coexistence). The blue curve is partitioned in this manner. Snapshots on the right correspond to the final configurations for the simulation runs in blue color.

particle separations $\left\langle u^{2}(t)\right\rangle \equiv\left\langle\left|\mathbf{u}_{i}(t)-\mathbf{u}_{j}(t)\right|^{2}\right\rangle$ as a function of time $t$, averaged over all pairs $(i, j)$ of particles that were nearest neighbors in the initial configuration [40]. For the present case, if in the long time limit $\left\langle u^{2}(t)\right\rangle$ saturated (on average) to a constant value much smaller than $R^{2}$ then the shell was assigned to be in a solid state. If, on the other hand, $\left\langle u^{2}(t)\right\rangle$ increases linearly in time until it reaches $R^{2}$ which is consistent with particle diffusion - then the shell was assigned to be in a fluid state. Finally, when plots of $\left\langle u^{2}(t)\right\rangle$ showed a random sequence of alternating time intervals of saturation and linear growth for a given simulation run with drastic variations between different runs then the shell was assigned to be in a fluid-solid coexistence state. Examples of these three cases are shown in Fig. 2 for $\gamma=10.6$. In the low-temperature solid state, with $\beta^{-1}=0.1$, the shell shape is spherical while in the coexistence regime, with $\beta^{-1}=0.7$, significant large-scale shape fluctuations are visible. The particle array still maintains local positional order but this has largely disappeared for $\beta^{-1}=1.6$ (fluid state). 


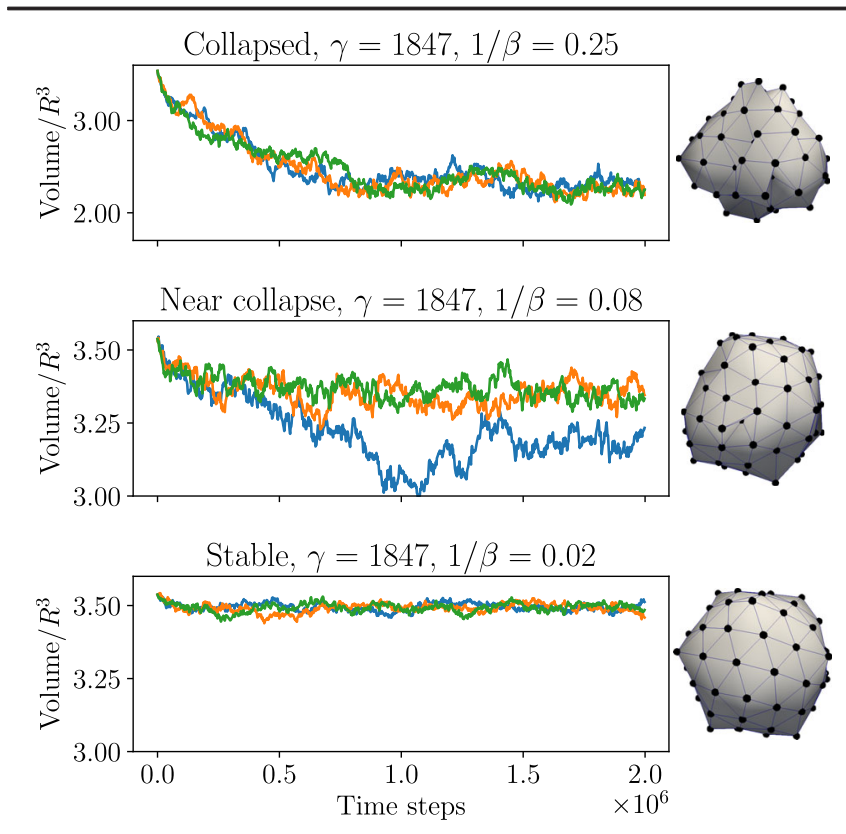

FIG. 3. Time traces of the shell volume for the FvK number $\gamma=1847$ and three different temperatures $\beta^{-1}$. Three separate simulation runs are shown in each case (orange, blue, and green, respectively). Bottom: for low temperatures $\left(\beta^{-1}=0.02\right)$ shell volumes reach a steady state after a small initial reduction. Top: for higher temperatures $\left(\beta^{-1}=0.25\right)$ the shell volumes drastically decrease indicating the collapse of shells. Middle: for $\beta^{-1}=$ 0.08 near the critical temperature for collapse, shell volumes exhibit large fluctuations and some shells undergo collapse (blue), while others remain stable (orange, green). Snapshots on the right correspond to the final configurations for the simulation runs in blue color.

The collapse of shells is a pronounced feature of the phase behavior of shells with larger values of the FvK number $\gamma$. Collapse was monitored by computing the volume of a continuous and differentiable surface that interpolates between the particle locations, which was constructed using the Loop shell subdivision method $[41,42]$. Figure 3(top) shows an example of irreversible collapse induced by thermal crumpling for $\gamma=1847$, as indicated by a drastic reduction in volume over time and the production of very irregular shell shapes. In Fig. 3(bottom) this simulation was repeated at a reduced temperature. After a small initial reduction, the shell volume reached a steady state [43]. The low-temperature shell shape is now icosahedral. In Fig. 3(middle), simulations were performed near the critical temperature for collapse. In this case shell volumes exhibit large fluctuations, with some shells undergoing a first-order-like collapse transition (blue time trace), while other shells remained stable over the simulated time interval.

By collecting simulation runs for different values of the $\beta^{-1}$ and $\gamma$ parameters, the phase plot of Fig. 4 was produced. The vertical bars indicate temperature intervals over which fluid-solid coexistence was observed following

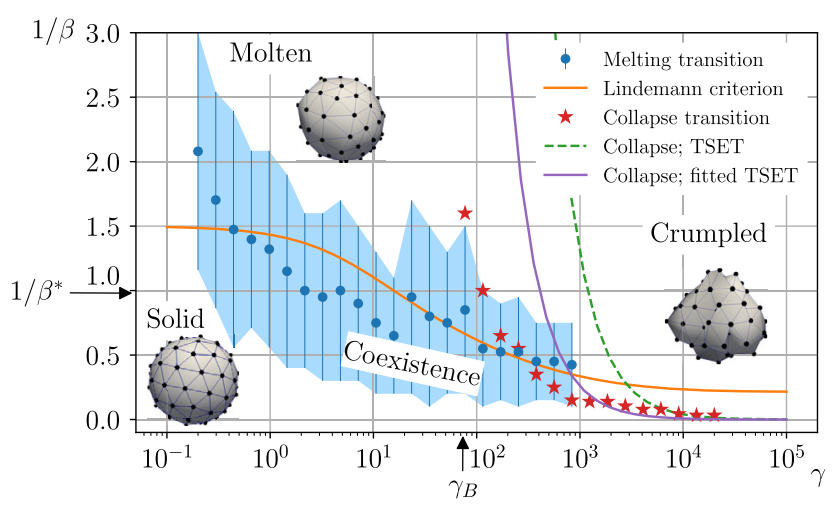

FIG. 4. Phase plot of the $N=72$ oriented particle system. The vertical axis is the dimensionless temperature $\beta^{-1}$ and the horizontal axis the FvK number $\gamma$. The KTHNY transition temperature $\beta^{*-1}$ for melting of flat sheets and the buckling threshold $\gamma_{B}$, where the minimum energy state goes from spherical [Fig. 2(bottom)] to polyhedral [Fig. 3(bottom)], are marked by arrows. The vertical blue bars mark intervals of phase coexistence separating solid and molten states. The orange line marks the melting temperature obtained from a combination of the Lindemann criterion and thin-shell elasticity theory (TSET). The red stars mark the onset of irreversible collapse obtained from the simulations. The green dashed line marks the onset of irreversible crumpling or collapse predicted by TSET and the purple line is a fit of the TSET scaling relation with an overall numerical scale factor to account for discreteness effects.

the criterion discussed above, with the solid blue dots indicating midpoints. The large coexistence interval for small $\gamma$ indicates that the melting transition should be first order on a rigid spherical surface, which is consistent with simulations of flat sheets of particles interacting via Morse potential [22]. The onset of irreversible collapse of shells for increasing temperature is indicated by orange triangles. Shells with $\gamma \gtrsim 10^{3}$ collapsed before the particle array could melt. On the other hand, shells with FvK numbers $\gamma$ in the range of $10^{2}-10^{3}$ would melt with increasing temperature before they collapsed.

To interpret the phase plot, we compared it with the thinshell elasticity theory (TSET) [44] in which a curved and stretched layer is assigned a bending and stretching energy given by

$$
\mathcal{H}=\int d s \frac{\kappa}{2} H^{2}+\int d s \frac{1}{2}\left(\lambda u_{i i}^{2}+2 \mu u_{i j}^{2}\right)
$$

Here, $\kappa$ is known as the Helfrich bending modulus of the layer, $H$ the local mean curvature, $\mu$ and $\lambda$ are twodimensional (2D) Lamé coefficients, while $u_{i j}$ is the strain tensor. The FvK number equals $\gamma=Y R^{2} / \kappa$ with $Y$ the corresponding 2D Young's modulus. For a flat sheet of point particles interacting via the Morse potential, $\lambda=\mu$ while $Y=(8 / 3) \mu$. In terms of the parameters of the OPS potential, $Y=4 \alpha^{2} V_{m} / \sqrt{3}$ and $\kappa=2 \sqrt{3} K$. 
According to TSET, $\gamma$ determines the ground-state shape of a thin elastic shell [39] such that for $\gamma$ less than a critical value $\gamma_{B}$ (the "buckling threshold") that is in the range of $10^{2}-10^{3}$, the shell has an approximately spherical shape [such as the spherical shape of Fig. 2(bottom)] while for $\gamma$ above that threshold, the ground-state shape is approximately polyhedral [such as the icosahedral shape of Fig. 3(bottom)]. As indicated in the phase plot, we find a buckling threshold around $\gamma_{B} \simeq 75$. The discrepancy between the computed and predicted values of the buckling threshold, which has been noted before [45], is a measure of the importance of discreteness effects. To apply TSET to melting, one can combine it with the Lindemann melting criterion (LMC), which states that melting occurs when the rms of the fluctuations of particle displacements exceeds a certain fraction of the equilibrium interparticle spacing $a$. The LMC is known to work well for melting on flat two-dimensional surfaces [40]. In the regime of harmonic fluctuations, the mean square $\left\langle\vec{u}^{2}\right\rangle$ of the in-plane fluctuations and the mean square $\left\langle f^{2}\right\rangle$ of the out-of-plane fluctuations can be shown to have the scaling form $\left\langle\vec{u}^{2}\right\rangle=$ $\left(k_{B} T / Y\right) G_{\infty}^{u}(\gamma)$ and $\left\langle f^{2}\right\rangle=\left(k_{B} T / Y\right) G_{\infty}^{f}(\gamma)$, respectively. The scaling functions $G_{\infty}^{u}(\gamma)$ and $G_{\infty}^{f}(\gamma)$ are discussed in the Supplemental Material [30], Sec. II. Formally, TSET theory corresponds to the limit of particle shells with $N$ large and $a$ small but with fixed radius $R \sim a N^{1 / 2}$. To include discreteness effects, we expanded the three displacement fields in terms of a series of spherical harmonics $\mathrm{Y}_{\ell, m}$ and demanded that the total number of out-of-plane modes $\mathcal{N}\left(\ell_{\max }\right)=\left(\ell_{\max }+1\right)^{2}$-with $\ell_{\max }$ the maximum value of the quantum number $\ell$-equals the number $N$ of particles minus two [46]. For a shell of $N=72$ particles $\ell_{\max }=7$ and $\ell_{\max }=8$ are reasonable choices (since 72 is in the interval between $8^{2}$ and $9^{2}$ ). The corrected scaling function has the same mathematical form as the TSET case but it is larger by an overall constant scale factor in the range of $10-100$, depending on the value of $\ell_{\max }$ : discreteness strongly amplifies the effects of thermal fluctuations. The resulting LMC melting temperatures $T_{m}(\gamma)=$ $T_{m}^{0} G_{N}^{u}(0) /\left[G_{N}^{u}(\gamma)+G_{N}^{f}(\gamma)\right]$ are plotted in Fig. 4 (orange line) with the melting temperature $T_{m}^{0}$ for $\gamma=0$ treated as a fitting parameter [47]. The resulting fit is reasonable for the range of $\gamma$ values over which melting was observed.

According to TSET, elastic shells with large $\gamma$ should undergo a collapse transition with increasing temperature [48-50]. Physically, this is due to the fact that crumpled shells have a much larger configurational space for shape fluctuations than (nearly) spherical shells so their entropy is much larger as well, while the volume of a crumpled shell with fixed area is correspondingly reduced. For larger $\gamma$, the enthalpic cost of crumpling the surface is diminished so a crumpling or collapse transition is to be expected. The collapse of a shell requires overcoming a free energy barrier that according to TSET vanishes when $k_{B} T \gamma^{1 / 2} / \kappa$ is about
$10^{2}$ (green dashed line in Fig. 4 [49,50]). For the crumpling or collapse transition that we observed (red stars), solid shells (but not liquid shells) roughly obeyed this scaling relation except that the value of $k_{B} T \gamma^{1 / 2} / \kappa$ had to be decreased by a factor of about 10 (purple line in Fig. 4). We interpret this as a discreteness effect similar to the one encountered for the melting transition. In the range of $\gamma \sim$ $10^{3}-10^{4}$ the fitted thin-shell elasticity theory (purple line) provides a reasonable estimate for the collapse transition. Small discrepancies with the molecular dynamics simulation (red stars) are attributed to thermally activated escape events over the energy barrier [50]. This is reflected in the middle panel of Fig. 3, where shell volumes exhibit large fluctuations, with some shells undergoing a first-order-like collapse transition (blue time trace), while other shells remained stable over the simulated time interval. In the range of $\gamma \sim 10^{2}-10^{3}$ there is a much larger discrepancy between the thin-shell elasticity and molecular dynamics simulations. For these values of $\gamma$ the collapse transition is in the same range as the melting transition. The unbound dislocation pairs that are forming during melting may significantly affect the collapse transition and this effect is not captured in the TSET.

As an example how the phase plot Fig. 4 could be applied to viral capsids, we compared the $N=72$ OPS with what is known about the phase properties of viral capsids having 72 capsomers. It should be noted that our phase plot corresponds to empty viral capsids, while the effect of osmotic pressure due to the packaged DNA would increase both the melting and collapse temperatures due to the suppression of radial fluctuations as discussed in [49]. In the Caspar-Klug classification of capsids, icosahedral shells with 72 capsomers are known as " $T=7$ " structures [4]. Medically important $T=7$ viruses are the human polyoma and papilloma unenvelopeded double-stranded DNA viruses, which both have a diameter of about $50 \mathrm{~nm}$ [51]. Because the polyoma and papilloma capsids are quite spherical, the value of $\gamma$ should, for these two cases, lie below the buckling threshold in Fig. 4. In contrast, two forms of the capsid of the $T=7$ thermostable DNA bacteriophage P23-45 with diameters of 66 and $82 \mathrm{~nm}$ are, respectively, weakly and strongly polyhedral [52]. This progression of $T=7$ shapes straddling the buckling threshold could be understood within TSET by noting that $\gamma$ scales as the square of the shell diameter. According to Fig. 4, in the relevant regime of $\beta^{-1} \simeq 1[5,6]$, capsids should be rather unstable in this part of the phase plot: prone both to melting and collapse. In actuality these viruses are known to be quite stable but this is because of a post-assembly maturation process, during which the capsid subunits are linked together by covalent bonds. The initial procapsids of the $T=7$ viruses, which are not yet bonded together, still could be thermodynamically unstable. Interestingly, the assembly of the procapsids of phages typically takes place on a preformed spherical scaffold that 
is disassembled during maturation [52]. It is very suggestive that one purpose of the scaffold is to prevent this instability. This could be checked experimentally by a study of the thermodynamic stability of self-assembling mutant $T=7$ empty shells for which maturation and/or scaffold formation is blocked. Instability of the procapsid of the related, but much larger, $T=13$ Herpes-Simplex virus should be even more pronounced.

We would like to thank Luigi Perotti, Jeff Eldredge and Bill Gelbart for useful discussions and the UCLA Mechanical and Aerospace Engineering Department for continued support. This work was supported by NSF through DMR Grants No. 1610384 and No. 1836404 , and the CAREER Award No. DMR-1752100.

[1] B. Alberts, Molecular Biology of the Cell (Garland Science, New York, 2017).

[2] T. S. Baker, N.H. Olson, and S. D. Fuller, Adding the third dimension to virus life cycles: Three-dimensional reconstruction of icosahedral viruses from cryo-electron micrographs, Microbiol. Mol. Biol. Rev. 63, 862 (1999).

[3] U. B. Sleytr, B. Schuster, E.-M. Egelseer, and D. Pum, S-layers: Principles and applications, FEM Micorbol. Rev. 38, 823 (2014).

[4] D. L. Caspar and A. Klug, Physical principles in the construction of regular viruses, in Cold Spring Harbor Symposia on Quantitative Biology, Vol. 27 (Cold Spring Harbor Laboratory Press, New York, 1962), pp. 1-24.

[5] P. Ceres and A. Zlotnick, Weak protein-protein interactions are sufficient to drive assembly of Hepatitis B Virus capsids, Biochemistry 41, 11525 (2002).

[6] S. Katen and A. Zlotnick, The thermodynamics of virus capsid assembly, Methods Enzymol. 455, 395 (2009).

[7] H. D. Nguyen, V. S. Reddy, and C. L. Brooks III, Invariant polymorphism in virus capsid assembly, J. Am. Chem. Soc. 131, 2606 (2009).

[8] This is due to kinetic trapping. For lower protein concentrations, the probability of kinetic trapping is reduced and interaction energies can be higher [9].

[9] E. C. Dykeman, P. G. Stockley, and R. Twarock, Solving a Levinthal's paradox for virus assembly identifies a unique antiviral strategy, Proc. Natl. Acad. Sci. U.S.A. 111, 5361 (2014).

[10] B. Bothner, A. Schneemann, D. Marshall, V. Reddy, J. E. Johnson, and G. Siuzdak, Crystallographically identical virus capsids display different properties in solution, Nat. Struct. Mol. Biol. 6, 114 (1999).

[11] J. A. Speir, B. Bothner, C. Qu, D. A. Willits, M. J. Young, and J.E. Johnson, Enhanced local symmetry interactions globally stabilize a mutant virus capsid that maintains infectivity and capsid dynamics, J. Virol. 80, 3582 (2006).

[12] A. J. Battisti, Structural studies of pleomorphic viruses, Ph.D. thesis, Purdue University, 2011.

[13] The Archaeal and Bunyaviridae families of viruses (which includes the Hanta viruses) [14-16] are important examples. The Gaussian curvature of some of the Archaeal pleomorphs evolves over time [14], indicating that they are in a fluidlike state $[17,18]$.

[14] D. Prangishvili, P. Forterre, and R. Garrett, Viruses of the Archaea: A unifying view, Nat. Rev. Microbiol. 4, 837 (2006).

[15] A. K. Överby, R. Pettersson, K. Grünewald, and J. Huiskonen, Insights into bunyavirus architecture from electron cryotomography of Uukuniemi virus, Proc. Natl. Acad. Sci. U.S.A. 105, 2375 (2008).

[16] M. K. Pietilä, S. Laurinavičius, J. Sund, E. Roine, and D. H. Bamford, The single-stranded DNA genome of novel archaeal virus Halorubrum pleomorphic virus 1 is enclosed in the envelope decorated with glycoprotein spikes, J. Virol. 84, 788 (2010).

[17] L. E. Perotti, S. Dharmavaram, W. S. Klug, J. Marian, J. Rudnick, and R. F. Bruinsma, Useful scars: Physics of the capsids of archaeal viruses, Phys. Rev. E 94, 012404 (2016).

[18] V. Vitelli, J. Lucks, and D. Nelson, Crystallography on curved surfaces, Proc. Natl. Acad. Sci. U.S.A. 103, 12323 (2006).

[19] P. L. Freddolino, A. S. Arkhipov, S. B. Larson, A. McPherson, and K. Schulten, Molecular dynamics simulations of the complete satellite tobacco mosaic virus, Structure 14, 437 (2006).

[20] D. Nelson and L. Peliti, Fluctuations in membranes with crystalline and hexatic order, J. Phys. (Paris) 48, 1085 (1987).

[21] R. Szeliski and D. Tonnesen, Surface Modeling with Oriented Particle Systems (ACM, New York, 1992), Vol. 26.

[22] S. I. Lee and S. J. Lee, Effect of the range of the potential on two-dimensional melting, Phys. Rev. E 78, 041504 (2008).

[23] H. Liu, C. Qu, J. E. Johnson, and D. A. Case, Pseudo-atomic models of swollen CCMV from cryo-electron microscopy data, J. Struct. Biol. 142, 356 (2003).

[24] J. E. Johnson and J. A. Speir, Quasi-equivalent viruses: A paradigm for protein assemblies, J. Mol. Biol. 269, 665 (1997).

[25] P. Ceres and A. Zlotnick, Weak protein-protein interactions are sufficient to drive assembly of Hepatitis B virus capsids, Biochemistry 41, 11525 (2002).

[26] A. Zlotnick, Are weak protein-protein interactions the general rule in capsid assembly?, Virology 315, 269 (2003).

[27] T. T. Nguyen, R. F. Bruinsma, and W. M. Gelbart, Elasticity theory and shape transitions of viral shells, Phys. Rev. E 72, 051923 (2005).

[28] W. H. Roos, R. Bruinsma, and G. J. L. Wuite, Physical virology, Nat. Phys. 6, 733 (2010).

[29] A. Y. Morozov, R. F. Bruinsma, and J. Rudnick, Assembly of viruses and the pseudo-law of mass action, J. Chem. Phys. 131, 155101 (2009).

[30] See the Supplemental Material at http://link.aps.org/ supplemental/10.1103/PhysRevLett.124.158101 for details of numerical methods and thin shell elasticity theory used in this work, which includes Refs. [31-38].

[31] C. Zhu, R. H. Byrd, P. Lu, and J. Nocedal, Algorithm 778: L-bfgs-b: Fortran subroutines for large-scale bound-constrained optimization, ACM Trans. Math. Softw. 23, 550 (1997). 
[32] W. Kabsch, A solution for the best rotation to relate two sets of vectors, Acta Crystallogr. A 32, 922 (1976).

[33] V. M. Bedanov, G. V. Gadiyak, and Y. E. Lozovik, On a modified Lindemann-like criterion for 2D melting, Phys. Lett. 109, 289 (1985).

[34] The CGAL Project, CGAL User and Reference Manual, 4th ed. (CGAL Editorial Board, 2019).

[35] V. Borrelli, F. Cazals, and J. M. Morvan, On the angular defect of triangulations and the pointwise approximation of curvatures, Comput. Aided Geom. Des. 20, 319 (2003).

[36] A. R. Singh, Study of zero and finite temperature response of discrete deformable surfaces, Ph.D. thesis, UCLA, 2018.

[37] K. Shoemake, Animating rotation with quaternion curves, in ACM SIGGRAPH Computer Graphics (ACM, New York, 1985), Vol. 19, pp. 245-254.

[38] Z. Zhang, H. T. Davis, and D. M. Kroll, Scaling behavior of self-avoiding tethered vesicles, Phys. Rev. E 48, R651 (1993).

[39] J. Lidmar, L. Mirny, and D. R. Nelson, Virus shapes and buckling transitions in spherical shells, Phys. Rev. E 68, 051910 (2003).

[40] H.-H. von Grünberg, P. Keim, K. Zahn, and G. Maret, Elastic Behavior of a Two-Dimensional Crystal Near Melting, Phys. Rev. Lett. 93, 255703 (2004).

[41] F. Cirak, M. Ortiz, and P. Schröder, Subdivision surfaces: A new paradigm for thin-shell finite-element analysis, Int. J. Numer. Methods Eng. 47, 2039 (2000).

[42] F. Feng and W. S. Klug, Finite element modeling of lipid bilayer membranes, J. Comput. Phys. 220, 394 (2006).

[43] Note that shape fluctuations always have to decrease the volume of shells, because the area of shells was kept fixed.

[44] H. S. Seung and D. R. Nelson, Defects in flexible membranes with crystalline order, Phys. Rev. A 38, 1005 (1988).
[45] A. R. Singh, L. E. Perotti, R. F. Bruinsma, J. Rudnick, and W. S. Klug, Ground state instabilities of protein shells are eliminated by buckling, Soft Matter 13, 8300 (2017).

[46] The total number of degrees of freedom is $3 N-6$, because translation and rotation are prevented.

[47] For small $\gamma$, the scaling function $G_{N}^{f}(\gamma)$ is proportional to $\gamma$ while $G_{N}^{u}(\gamma)$ is constant. The reduction of the melting temperature with increasing $\gamma$ is thus dominated by the out-of-plane fluctuations.

[48] J. Paulose, G. A. Vliegenthart, G. Gompper, and D. R. Nelson, Fluctuating shells under pressure, Proc. Natl. Acad. Sci. U.S.A. 109, 19551 (2012).

[49] A. Košmrlj and D. R. Nelson, Statistical Mechanics of Thin Spherical Shells, Phys. Rev. X 7, 011002 (2017).

[50] L. Baumgarten and J. Kierfeld, Buckling of thermally fluctuating spherical shells: Parameter renormalization and thermally activated barrier crossing, Phys. Rev. E 97, 052801 (2018).

[51] D. M. Belnap, N. H. Olson, N. M. Cladel, W. W. Newcomb, J. C. Brown, J. W. Kreider, N. D. Christensen, and T. S. Baker, Conserved features in papillomavirus and polyomavirus capsids, J. Mol. Biol. 259, 249 (1996).

[52] O. W. Bayfield, E. Klimuk, D. C. Winkler, E. L. Hesketh, M. Chechik, N. Cheng, E. C. Dykeman, L. Minakhin, N. A. Ranson, K. Severinov et al., Cryo-em structure and in vitro dna packaging of a thermophilic virus with supersized $t=7$ capsids, Proc. Natl. Acad. Sci. U.S.A. 116, 3556 (2019).

Correction: The previously published Figure 2 was converted improperly during the production process and its rendition has been corrected. 